\title{
Obliterative bronchiolitis: varying presentations and clinicopathological correlation
}

\author{
K.D. Markopoulou*,§, C.D. Cool*,+, T.L. Elliot ${ }^{\#,+}$, D.A. Lynch ${ }^{\#,+}$, J.D. Newell Jr ${ }^{\#,+}$, V.A. Hale ${ }^{\#,+}$, \\ K.K. Brown ${ }^{+}$, M.I. Schwarz ${ }^{\uparrow,+}$, R.M. Tuder*
}

Obliterative bronchiolitis: varying presentations and clinicopathological correlation. K.D. Markopoulou, C.D. Cool, T.L. Elliot, D.A. Lynch, J.D. Newell Jr, V.A. Hale, K.K. Brown, M.I. Schwarz, R. M. Tuder. (C) ERS Journals Ltd 2002.

ABSTRACT: In obliterative bronchiolitis, inflammation and fibrosis lead to narrowing or occlusion of bronchiolar lumina.

To determine how bronchiolar structural alterations relate to lung physiology, 19 patients with a pathological diagnosis of obliterative bronchiolitis were studied. The bronchiolar inflammatory and fibrotic features were correlated to the clinical presentation, and lung function tests.

Eleven patients demonstrated airflow limitation, one had a restrictive pattern and one had a mixed pattern, two had isolated gas trapping, but four had normal spirometry. Mild-to-moderate bronchiolar inflammation was invariably present. It involved $60 \%$ of bronchioles subepithelially and $54 \%$ in the adventitia. Subepithelial fibrosis was observed in 15 patients and adventitial in 12. Adventitial bronchiolar inflammation correlated with forced expiratory volume in one second and forced vital capacity and inversely correlated with residual volume. Subepithelial fibrosis inversely correlated with subepithelial and adventitial inflammation. High-resolution computed tomography in 10 patients revealed inspiratory (five out of 10) and expiratory air trapping (five out of five), ground glass opacities (seven out of 10), bronchial wall thickening (five out of 10), bronchiectasis (two out of 10) and centrilobular nodules (two out of 10).

The present study suggests that inflammation and fibrosis occurs in bronchioles at different time points in the disease process, or that there is no transition between these types of pathology in the same patient. No correlation was observed between the degree of bronchiolar fibrosis and the degree of airflow limitation.

Eur Respir J 2002; 19: 20-30.

\begin{abstract}
Depts of *Pathology, ${ }^{\#}$ Radiology, and Division of Respiratory Sciences and Critical Care Medicine, University of Colorado Health Sciences Center, Denver, Colorado, USA. ${ }^{+}$National Jewish Medical and Research Center, Denver, Colorado, USA. ${ }^{8} 1$ st Pulmonology Dept, George Papanikolaou Hospital, Thessaloniki, Greece.
\end{abstract}

Correspondence: K. Markopoulou, Patriarchou Ioakeim 12, Thessaloniki, Greece.

Fax: 30310277742

Keywords: Constrictive bronchiolitis fibrosis

inflammation

pathology

spirometry

Received: June 262001

Accepted after revision July 12001

This study was supported by the National Heart Lung and Blood Institute Specialized Center of Research Grant H1-27353.
Chronic inflammation and luminal compromise of the bronchioles has been recognized as a cause of respiratory disease since the beginning of the 20th century. However, the terminology and clinical presentation of bronchiolar diseases still causes confusion. LANGE [1], in 1901, introduced the term bronchiolitis obliterans to describe a case in which the bronchiolar lumens were obstructed by plugs of granulation tissue originating from the bronchiolar wall. In 1929, the term bronchiolitis fibrosa obliterans was used for similar findings [2], whereas in 1966 it referred to lesions consisting of concentric rings of fibrotic tissue in the wall of the airways [3]. MACKLEM et al. [4] in 1971, introduced the term chronic obstructive small airways disease describing seven patients with airflow limitation, who had chronic bronchiolar inflammation, with variable degrees of airway wall fibrosis. The term constrictive bronchiolitis was coined by Gosink et al. [5] in 1973, to describe a peribronchiolar and mural infiltrate of mononuclear cells and granulation tissue, resulting in a compromise of the bronchiolar lumen. TURTON et al. [6] 8 yrs later, coined the term cryptogenic obliterative bronchiolitis to characterize cases with otherwise unexplained progressive airflow obstruction. These patients had forced expiratory volume in one second (FEV1) $<60 \%$ predicted, no bronchodilator response or peak expiratory flow variability and no history of episodic wheezing, atopy, chronic bronchitis, emphysema or known causes of chronic airflow obstruction. In 1987, GUERRY-ForCE et al. [7] studied nine patients with histologically proven inflammation of small airways and described patients with both obstructive and restrictive spirometric patterns. Two years later, KINDT et al. [8] described bronchiolitis in 16 adult patients who had histological lesions of chronic bronchiolitis with spirometric patterns of obstruction, restriction or mixed obstruction and restriction. EDWARDS et al. [9] in 1992, used the term chronic transmural bronchiolitis to describe chronic bronchiolar inflammation in nine patients with different underlying diseases and obstructive or restrictive spirometric patterns. KRAFT et al. [10], in 1993, reported four females with a histological diagnosis of constrictive bronchiolitis, one of which had normal spirometry and one of which had a mixed obstructive 
and restrictive pattern of disease. From these reports, it is clear that the disease spectrum associated with histological evidence of bronchiolar inflammation and fibrosis is varied, comprising cases with obstructive, restrictive or normal pattern of spirometry and diverse clinical and radiological presentation. Descriptive terminology is both evolving and continuously being revisited. The term chosen for use in this paper is obliterative bronchiolitis, because it evokes best the essence of luminal narrowing, which is caused by both inflammation and fibrosis. Obliterative bronchiolitis occurs in a number of clinical settings. These include infection, inhalational injury, bone-marrow transplantation, lung or heart-lung transplantation, drug toxicity, or in the context of a collagen-vascular disease. There are also idiopathic cases [11].

Bronchiolar changes in obliterative bronchiolitis may vary considerably. They can be subtle or show complete luminal obliteration. The histological abnormalities include submucosal and adventitial scarring, smooth-muscle hypertrophy, distortion of the airway lumen, bronchiolectasis, mucostasis and bronchiolarization of adjacent alveolar spaces. Inflammatory changes, submucosal, mural or peripheral (cellular bronchiolitis), are also common. In a given patient, any constellation of these findings may occur. The pathology does not always correlate with the functional status of the patients. Moreover, histological evidence for the disease may be present without physiological airflow limitation [12].

Presently, there is no clear understanding of how the diverse structural alterations relate to lung physiology, and whether a predominantly inflammatory component, compared to a dominant pattern of collagen deposition, impacts differently on the presentation and evolution of the bronchiolar disease.

This study was undertaken to test the hypothesis that the degree of inflammation or fibrosis in the small airways correlates with the clinical presentation in patients with the pathological diagnosis of obliterative/constrictive bronchiolitis.

\section{Material and methods}

\section{Study subjects}

A search of the "Pathology Database" from the University of Colorado Health Sciences Center and the "Interstitial Lung Disease Database" of the National Jewish Medical and Research Center was carried out. All cases involving a pathological diagnosis of obliterative or constrictive bronchiolitis/ bronchiolitis obliterans/chronic bronchiolitis, proven by either open lung biopsy, video-assisted thoracoscopic biopsy, or examination of explanted lung, for the years 1993-2000, were reviewed. Inclusion criteria were the presence of luminal narrowing caused by chronic bronchiolar inflammation, increased bronchiolar collagen deposition, or both. Cases which developed following bone-marrow, lung or heartlung transplantation were excluded. Patients with a histological diagnosis of respiratory bronchiolitis interstitial lung disease and diffuse panbronchiolitis were also excluded.

\section{Study design}

Medical charts were abstracted and lung function tests prior to the biopsy were tabulated. Highresolution computed tomography (HRCT) scans of the chest, when available, were evaluated. Sections stained with haematoxylin and eosin were reviewed for a detailed description of findings, and scored for bronchiolar inflammation. Sections stained for collagen and smooth-muscle actin were examined for the presence of fibrosis. A control group consisted of 10 autopsy cases from the University of Colorado Hospital with normal lung histology.

\section{Chart abstraction}

Medical charts were abstracted for demographical information, smoking history, symptomatology, occupational or environmental exposure, past medical history, medications, and collagen vascular diseases serology. Nineteen patients (five male and 14 female) were included. All were Caucasian except for two Hispanics. Their median age was 45 yrs (confidence interval (CI) 36.5-54.3). Ten were nonsmokers, six were former smokers with a mean pack-yr history of 19.9 (range 2.5-60) and three were current smokers with a mean pack-yr history of 32 (range 6-14). Almost all of the patients had cough and dyspnoea prior to biopsy. Other symptoms included fever and chills in patient 14 , myalgias and arthralgias in patient 17, anaemia, weight loss and arthralgias in patient 18 and haemoptysis in patient 19. Sputum production was minimal except for one individual, who was a smoker. The symptoms and their duration prior to biopsy are summarized in table 1 .

\section{Respiratory function}

Eleven patients had an obstructive pattern by spirometric evaluation and nine of these had a FEV1 $<60 \%$ predicted. One patient had a restrictive pattern and one a mixed obstructive-restrictive pattern. Two patients had evidence of gas trapping, but without airflow limitation. Four patients had normal spirometry at the time of the biopsy. The spirometric values for the entire cohort are summarized in table 2 and for the subgroup with an obstructive pattern in table 3 .

\section{Onset of disease and exposure history}

The time of disease onset could not be clearly identified in thirteen patients and symptoms gradually worsened. Three had well documented toxic-fume exposures prior to symptoms. Patient 15 was exposed to ammonia, nitrogen oxide $\left(\mathrm{NO}_{2}\right)$, carbon dioxide $\left(\mathrm{CO}_{2}\right)$ and carbon monoxide $(\mathrm{CO})$ while fighting a fire at a photography processing laboratory, and patient 8 
Table 1. - Patient characteristics, symptoms and their duration

\begin{tabular}{|c|c|c|c|c|c|c|c|c|c|c|c|c|c|}
\hline $\begin{array}{l}\text { Subject } \\
\text { no. }\end{array}$ & Sex & $\begin{array}{c}\text { Age } \\
\text { yrs }\end{array}$ & Smoking & $\begin{array}{c}\text { Pack- } \\
\text { yrs }\end{array}$ & $\begin{array}{l}\text { Years } \\
\text { since } \\
\text { quitting }\end{array}$ & Cough & $\begin{array}{l}\text { Cough } \\
\text { duration } \\
\text { months }\end{array}$ & Sputum & $\begin{array}{c}\text { Sputum } \\
\text { duration } \\
\text { months }\end{array}$ & Dyspnoea & $\begin{array}{c}\text { Dyspnoea } \\
\text { duration } \\
\text { months }\end{array}$ & Wheeze & $\begin{array}{l}\text { Wheeze } \\
\text { duration } \\
\text { months }\end{array}$ \\
\hline 1 & $\mathrm{M}$ & 57 & FS & 7 & 19 & & & & & + & 31 & & \\
\hline 2 & $\mathrm{~F}$ & 21 & NS & & & + & 2 & & & + & 0.25 & & \\
\hline 3 & $\mathrm{~F}$ & 45 & NS & & & + & 9 & + & 7 & + & 9 & & \\
\hline 4 & $\mathrm{~F}$ & 50 & $\mathrm{FS}$ & 14 & 3 & + & 84 & + & 84 & + & 48 & + & 84 \\
\hline 5 & $\mathrm{~F}$ & 51 & CS & 20 & & + & 4 & & & + & 4 & + & 4 \\
\hline 6 & $\mathrm{~F}$ & 36 & NS & & & + & 24 & & & + & 24 & & \\
\hline 7 & $\mathrm{~F}$ & 44 & CS & 22 & & + & 2 & + & & + & 72 & & \\
\hline 8 & $\mathrm{M}$ & 38 & $\mathrm{CS}$ & 6 & & + & 10 & & & + & 10 & + & 10 \\
\hline 9 & $\mathrm{~F}$ & 38 & NS & & & + & 15 & + & 15 & + & 15 & + & 15 \\
\hline 10 & $\mathrm{~F}$ & 58 & FS & 2.5 & 32 & & & & & + & 12 & & \\
\hline 11 & $\mathrm{M}$ & 50 & FS & 60 & 2 & & & & & + & 60 & & \\
\hline 12 & $\mathrm{~F}$ & 52 & NS & & & + & 12 & & & + & 12 & & \\
\hline 13 & $\mathrm{M}$ & 36 & FS & 16 & 4 & + & 0.5 & & & + & 7 & & \\
\hline 14 & $\mathrm{~F}$ & 57 & NS & & & + & 24 & & & & & & \\
\hline 15 & $\mathrm{M}$ & 34 & NS & & & + & 4 & & & + & 4 & + & 4 \\
\hline 16 & $\mathrm{~F}$ & 62 & NS & & & + & 6 & + & 4 & + & 6 & & \\
\hline 17 & $\mathrm{~F}$ & 44 & NS & & & + & 12 & + & 12 & + & 16 & + & 12 \\
\hline 18 & $\mathrm{~F}$ & 35 & NS & & & + & 288 & + & 60 & + & 60 & + & 60 \\
\hline 19 & $\mathrm{~F}$ & 55 & FS & 20 & 8 & & & & & + & 72 & + & 12 \\
\hline
\end{tabular}

M: male; F: female; FS: former smoker; NS: never-smoker; CS: current smoker; +: positive for given symptom.

was exposed to polymethylene polyphenol isocyanate (polymeric diphenylmethane disocyanate product) for $3 \mathrm{~h}$, without respiratory protection, while performing maintenance work at a plastics factory. Patient 6 had an unspecified fume exposure. Upper respiratory tract infection or flu-like syndrome preceded symptoms in patients 2 and 6 . Patients 13 and 14 reported an acute onset of disease, but without any definite precipitating event.

A history of environmental exposure was obtained from six patients. Patients 6 and 9 had pet birds in the home, patient 17 had worked in silos, and patient 13 had worked in a factory where ammonia was periodically used and had also worked and lived near silos. Patient 14 had possible mould exposure, secondary to water damage in the house, as well as talc inhalation. The talc was identified in the lung biopsy and was thought to originate from carpets and air ducts in a newly constructed home. Patient 11 had been a coal miner. Three patients were suspected of

Table 2. - Lung function tests

\begin{tabular}{|c|c|c|c|c|c|c|c|c|}
\hline Patient no. & FEV1 & FVC & FEV1/FVC & TLC & RV & $D \mathrm{~L}, \mathrm{CO} / V \mathrm{~A}$ & MEF & $\mathrm{B} / \mathrm{D}$ \\
\hline 1 & 14 & 32 & 35 & 105 & 200 & 100 & & \\
\hline 2 & 49 & 53 & 79 & & & & & \\
\hline 3 & 17 & 49 & 38 & & & & & \\
\hline 4 & 72 & 73 & 80 & 96 & 172 & 99 & 86 & 9 \\
\hline 5 & 53 & 61 & 69 & & & 116 & & 8 \\
\hline 6 & 28 & 57 & 41 & 124 & 403 & 119 & 11 & 9 \\
\hline 7 & 14 & 33 & 41 & 132 & 360 & 61 & 5 & 40 \\
\hline 8 & 84 & 83 & 76 & 85 & 108 & 91 & 70 & 16 \\
\hline 9 & 82 & 79 & 88 & 89 & 106 & 80 & 85 & 12 \\
\hline 10 & 70 & 90 & 60 & 128 & 262 & 95 & 48 & 9 \\
\hline 11 & 23 & 49 & 33 & 127 & 389 & 53 & 17 & 1 \\
\hline 12 & 52 & 62 & 68 & 99 & 163 & 84 & & 11 \\
\hline 13 & 21 & 28 & 56 & 66 & 122 & & 16 & \\
\hline 14 & 63 & 64 & 76 & 97 & 165 & 109 & 82 & 14 \\
\hline 15 & 84 & 92 & 72 & 98 & 76 & 100 & 91 & 7 \\
\hline 16 & 76 & 61 & 92 & 98 & 98 & 87 & 113 & \\
\hline 17 & 79 & 80 & 81 & 98 & 135 & 100 & & \\
\hline 18 & 23 & 66 & 30 & 135 & 349 & 61 & 6 & \\
\hline 19 & 38 & 61 & 48 & 90 & 134 & 118 & 20 & -1 \\
\hline Median & 52 & 61 & 68 & 98 & 164 & 97 & 48 & \\
\hline CI & $24-75$ & $53-77$ & $43-78$ & $93-125$ & $115-305$ & 82-104 & $15-87$ & \\
\hline
\end{tabular}

All data is presented as \% predicted. FEV1: forced expiratory volume in one second; FVC: forced vital capacity; TLC: total lung capacity; RV: residual volume; $D \mathrm{~L}, \mathrm{CO} / V \mathrm{~A}$ : Diffusing capacity for carbon monoxide corrected for alveolar volume; MEF: midexpiratory flow (between $25 \%$ and $75 \%$ of $\mathrm{FVC}$ ); B/D: percentage change in FEV1 after $\beta_{2}$-agonist inhalation. 
Table 3.-Lung function tests for the subgroup with obstructive pattern and forced expiratory volume in one second $\left(F E V_{1}\right)<60 \%$ predicted

\begin{tabular}{lc}
\hline Vaiable & Data \\
\hline Subjects n & 9 \\
FEV1 & $28(21.5-41.5)$ \\
FVC & $57(49-61.2)$ \\
FEV1/FVC & $41(34.5-57.5)$ \\
TLC & $124(100.5-130.7)$ \\
RV & $349(172.2-381.7)$ \\
$D L, C O / V A$ & $92(61-117)$ \\
MEF & $11(5.7-17.7)$ \\
\hline
\end{tabular}

Data are presented as \% pred median (confidence interval). FVC: forced vital capacity; TLC: total lung capacity; RV: residual volume; $D \mathrm{~L}, \mathrm{CO} / V \mathrm{~A}$ : diffusing capacity for carbon monoxide corrected for alveolar volume; MEF: midexpiratory flow between $25 \%$ and $75 \%$ of FVC.

having hypersensitivity pneumonitis because of the presence of poorly formed granulomas (patient 9) or isolated giant cells (patients 6 and 10) on lung biopsy. As mentioned, patients 6 and 9 had pet birds at home. No exposure was identified in patient 10 .

\section{Serology}

Antinuclear antibodies were measured in seven patients. One patient (patient 19) without systemic disease had a speckled pattern (1:160). Patient 18 was positive (1:80 speckled pattern), and also had positive antimyeloperoxidase titre (perinuclear antineutrophil cytoplasmic antibodies) of 1:10,240. Two patients had positive rheumatoid factors, patient 3 with active rheumatoid arthritis and patient 6 without joint disease.

\section{Past medical history}

Six patients (patients 3, 5, 7, 9, 18, 19) had a prior clinical diagnosis of asthma with progressive deterioration of lung function, despite therapy with inhaled and systemic corticosteroids. Bronchodilator response was available for eight patients who did not have a clinical diagnosis of asthma. Three of these had positive responses $(>10 \%)$. Patient 12 had chronic renal failure and had been on home dialysis for 25 yrs. Patient 18, during the course of her respiratory disease, had developed migratory arthralgias, anaemia, weight loss and an antineutrophil cytoplasmic antibody directed against myeloperoxidase (MPO-ANCA) titre of $1: 10,240$. Only one patient (patient 3) had rheumatoid arthritis, and had been treated with gold, plaquenil, methotrexate and sulphasalazine in the past. Five female patients (patients $5,7,10,12,19)$ had undergone hysterectomy, one (patient 5) for uterine neoplasm. Four patients (patients $9,10,15,19)$ had a history of multiple pneumonias. Six patients (patients 4, 6, 7, 8, 15, 18) had received oral corticosteroids for variable periods of time prior to lung biopsy.

\section{Pathology}

All available sections from each case were reviewed three times (twice by pulmonary pathologists). If another significant histological finding, such as interstitial inflammation, prominent fibrosis or intraalveolar exudates (organizing pneumonia, bronchiolitis obliterans organizing pneumonia, diffuse alveolar damage/acute interstitial pneumonia) was present, the case was excluded. Two slides from each of the final 19 cases, that had the greatest sample of bronchioles, were selected for further evaluation.

Additional serial sections from each case were prepared from the corresponding paraffin blocks. These slides were double-stained, first with an antibody directed against smooth muscle-specific actin to visualize the smooth muscle component of the bronchial wall, and second with picric acid-fast green stain (Pfalz and Bauer, Waterbury, CT, USA) for identification of the bronchial wall collagen.

\section{Immunohistochemistry for smooth muscle actin}

Sections were stained for smooth-muscle actin as described by Cool et al. [13].

\section{Staining for collagen}

After immunostaining for smooth muscle-specific actin, the sections were immersed for $30 \mathrm{~min}$ in a solution of $100 \mathrm{~mL}$ of saturated aqueous solution of picric acid with $0.1 \mathrm{~g}$ of fast-green FCF (Pfalz and Bauer, Waterbury, CT, USA) and 0.1 gm of Sirius-red F3BA (Pfalz and Bauer, Waterbury, CT, USA). The sections were then mounted with a medium and cover slipped.

\section{Examination for bronchiolar inflammation}

Two haematoxylin and eosin sections were scored for each case. Chronic inflammation was classified as either absent or present, with either a subepithelial, adventitial or both subepithelial and adventitial distribution (figs. 1-3). The degree of inflammation was scored semiquantitatively as minimal grade (1), moderate grade (2) or severe grade (3) for each bronchiole examined. The mean value for each anatomical type of inflammation, in each case, was derived by counting all bronchiolar structures present on both slides. A percentage was derived by counting inflamed bronchioles relative to the total number of bronchioles. An inflammation score (IS) was calculated by multiplying the degree of inflammation by the percentage of involved bronchioles. The maximum IS would be 300 for any patient (maximum inflammation score $3 \times 100 \%$ of bronchioles).

\section{Examination for airway wall fibrosis}

The collagen stain indicated five patterns of bronchiolar wall fibrosis: 1) subepithelial deposition 


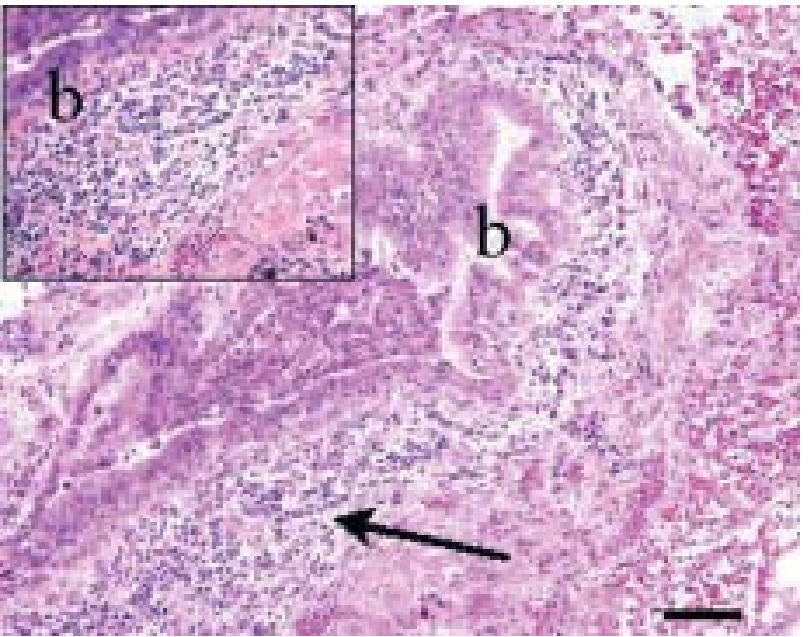

Fig. 1.-Terminal bronchiole with marked subepithelial inflammation resulting in displacement of the lining epithelium towards the lumen (arrow and inset). Internal scale bar=82 microns.

(SF), usually in the form of thickening of the basement membrane; 2) adventitial deposition (AF), appearing as a cuff surrounding the bronchiole; 3) a combination of SF and AF; 4) minimal collagen deposition (MC), consisting of simple strands of collagen intermingled with inflammatory and resident cells; and 5) a thin layer of collagen in the basement membrane, which was indistinguishable from that present in the normal control bronchioles (figs. 4-8). Each bronchiole was examined for the presence or absence of one of the fibrotic patterns, and the percentage of bronchioles with SF, AF, MC was calculated.

\section{High-resolution computed tomography}

Three radiologists reviewed the scans, without knowledge of the patients' clinical histories, for the presence of bronchial-wall thickening, bronchiolectasis,

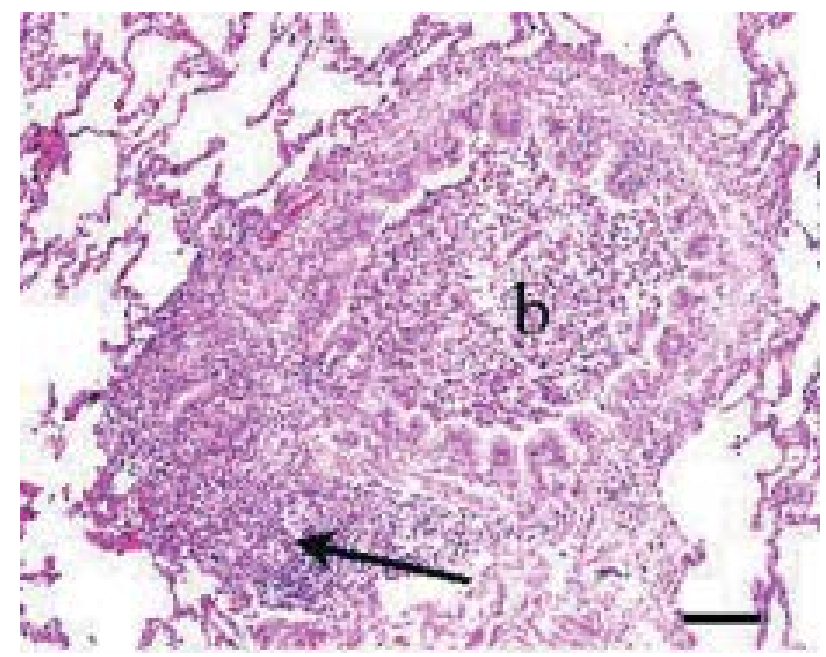

Fig. 2. - Example of adventitial bronchiolar inflammation, with a nodular clustering of lymphoid cells (arrow). Internal scale bar=82 microns.

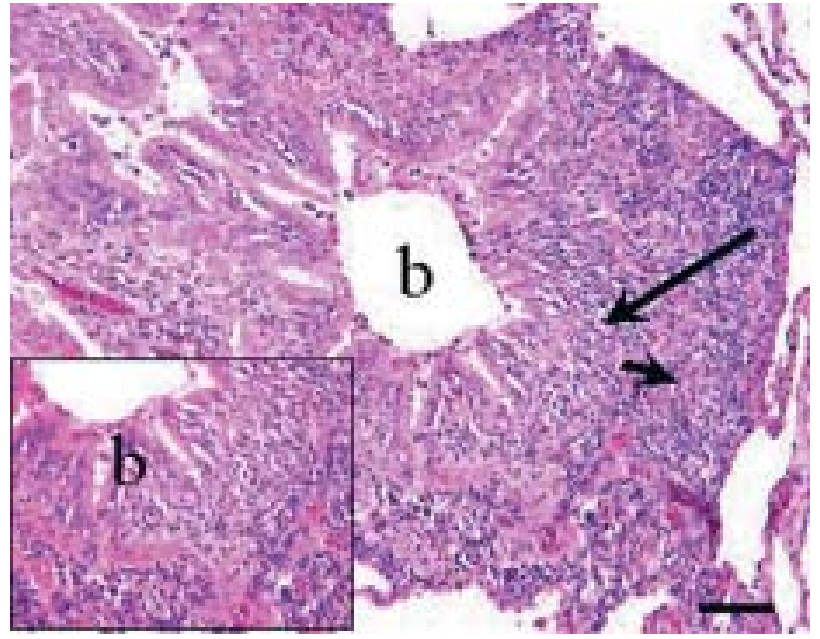

Fig. 3-Example of severe mural inflammation in terminal bronchiole involving both the subepithelial (long arrow and inset) and adventitial (short arrow) regions. Note the almost complete obliteration of the bronchiolar lumen by the thickened bronchial mucosa (b) (magnification). Internal scale bar=82 microns.

tree-in-bud, centrilobular nodules, bronchiectasis, ground-glass opacities, consolidation, emphysema, inspiratory and expiratory air-trapping. In cases of discrepancies, the scans were re-examined and a consensus decision was reached.

\section{Statistical analysis}

All data were graded on a continuous scale. Spearman's rank correlation was used to examine the correlations between symptoms, spirometric values, pathological findings and HRCT findings. Kruskall-Wallis one-way analysis of variance was used for comparisons in spirometric values and pathological findings between current, former and never-smokers. The Mann-Whitney $U$ test was used for comparisons in histological findings between

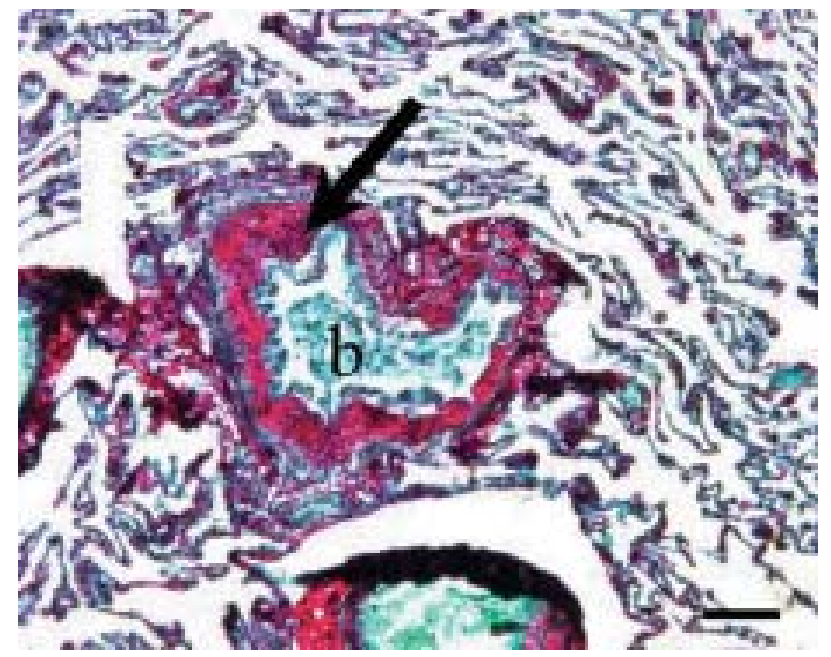

Fig. 4.-Example of subepithelial fibrosis (arrow) resulting in distortion of bronchiolar lumen. Internal scale bar $=82$ microns. 


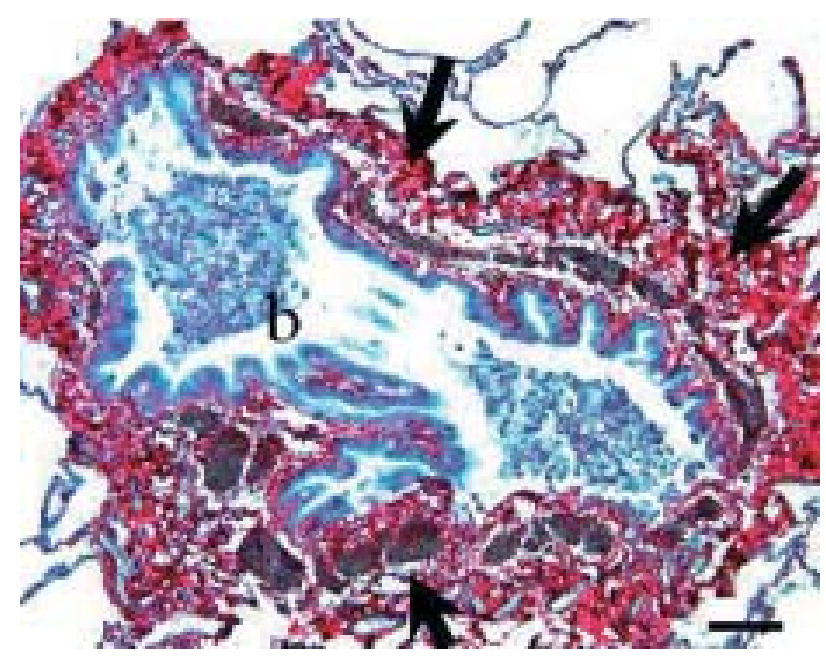

Fig. 5.-Example of adventitial fibrosis (top arrows). The bronchiolar smooth muscle layer is clearly discerned (bottom arrow) immunostained with antibody against smooth muscle actin. Internal scale bar $=82$ microns.

patients with normal and obstructive patterns of spirometry.

\section{Results}

\section{Histological findings}

All cases had evidence of luminal narrowing. The next most common finding was chronic inflammation of the bronchioles. Descriptions of the histological lesions are summarized in table 4 , and the original pathological diagnoses in table 5 .

\section{Bronchiolar inflammation}

A median of 10 (CI 9-12.5) bronchioles was analysed for each patient. Inflammation was generally

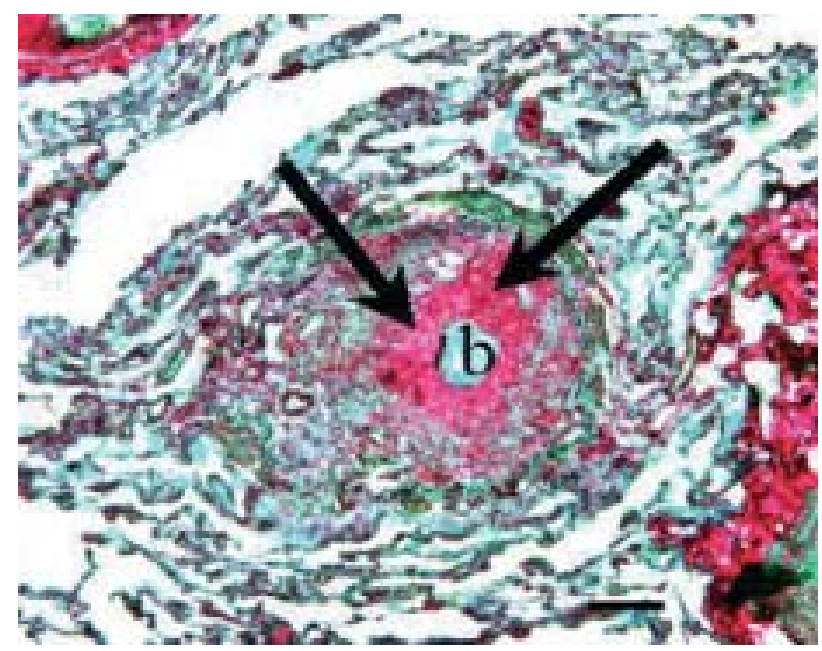

Fig. 6.-Advanced mural fibrosis (arrows) resulting in almost complete obliteration of bronchiolar lumen (b). Internal scale bar $=82$ microns

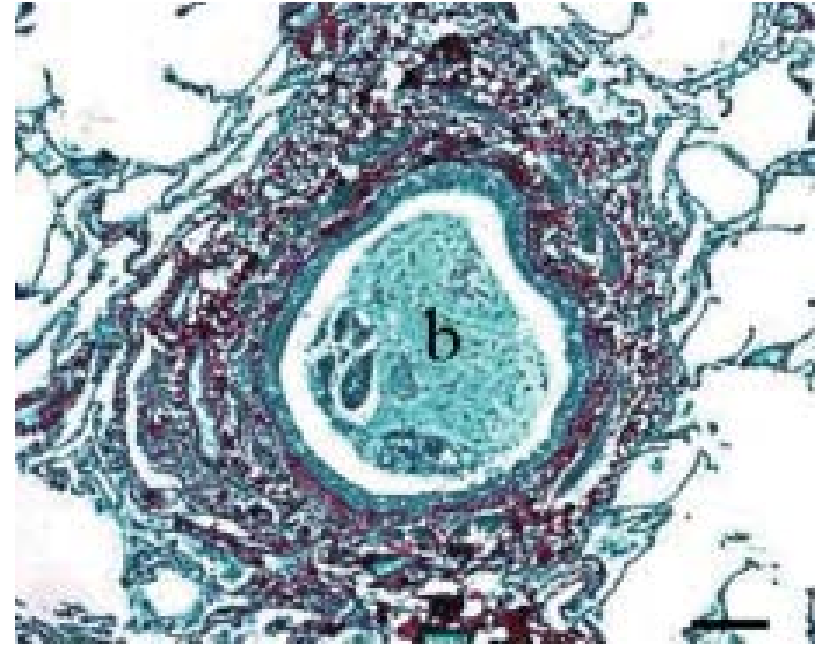

Fig. 7.-Minimal collagen deposition in the wall of a terminal bronchiole as assessed by picrosirius red. Internal scale bar=82 microns.

mild to moderate and involved approximately half the bronchioles examined. The results are summarized in table 6 .

\section{Collagen deposition}

A median of 16 bronchioles (CI 11.3-23) was studied for each patient. SF of collagen was observed in 15 of the 19 cases. AF was noted in 12 patients. In both subepithelial and adventitial bronchiolar regions, the collagen deposition affected a limited number of bronchioles (medians of $29 \%$ subepithelially and 16\% in the adventitia). Results are summarized in table 7.

\section{Inflammation and collagen deposition}

SF inversely correlated with the extent of subepithelial inflammation $(\% \mathrm{SI})\left(\mathrm{r}_{\mathrm{s}}=-0.55, \mathrm{p}=0.01\right)$, the

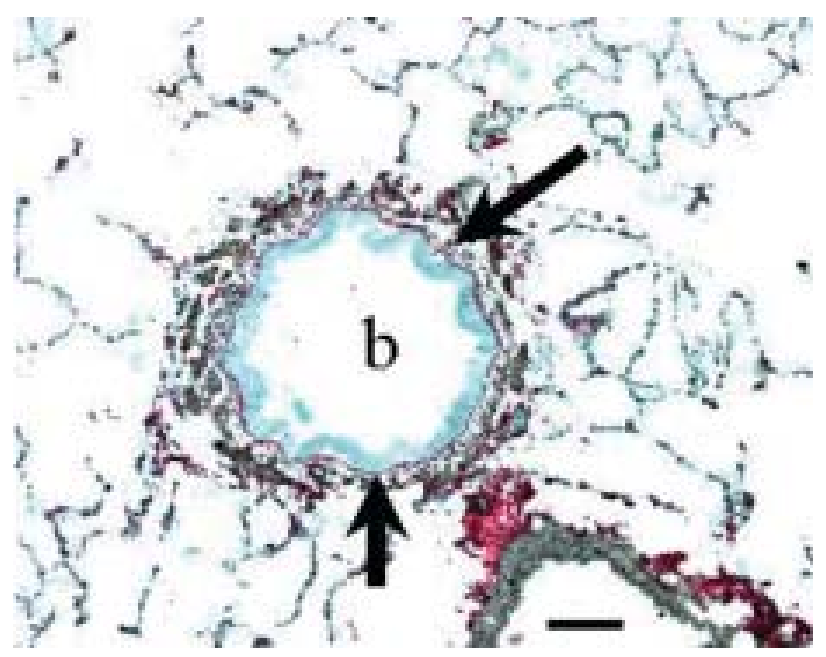

Fig. 8.-Example of a normal terminal bronchiole with a thin collagen layer stained with picrosirius red (arrow). Internal scale bar $=51$ microns. 
Table 4.-Detailed histological findings

\begin{tabular}{l}
\hline Lesion \\
\hline Bronchioles \\
Acute inflammation \\
Chronic inflammation \\
Eosinophils \\
Goblet cell hyperplasia \\
Basement membrane \\
thickening \\
Submucosal oedema \\
Smooth muscle hyperplasia \\
Inspissated mucus \\
Bronchiolodilatation \\
Bronchiolar wall destruction \\
Luminal obliteration \\
Lymphoid hyperplasia \\
Respiratory bronchiolitis \\
Alveoli \\
Bronchiolarization* \\
Alveolar wall fibrosis \\
Alveolar wall inflammation \\
Emphysema \\
Alveolar macrophages \\
Haemorrhage \\
BOOP \\
Granulomas \\
Giant cells
\end{tabular}

*: extension of bronchiolar type epithelium to centrilobular alveolar septae that have been modified by chronic inflammation with or without scarring. BOOP: bronchiolitis obliterans organizing pneumonia.

subepithelial inflammation score (SIS) $\left(\mathrm{r}_{\mathrm{s}}=-0.55, \mathrm{p}=0.01\right)$ and the grade of adventitial inflammation (AIG) $\left(\mathrm{r}_{\mathrm{s}}=\right.$ $-0.51, \mathrm{p}=0.02)$. The extent of minimal collagen deposition inversely correlated with $\mathrm{AF}(\mathrm{r}=-0.54, \mathrm{p}=0.01)$.

\section{Histopathological findings and spirometric values}

The adventitial inflammation score (AIS) correlated with $\mathrm{FEV} 1 \%$ pred $\left(\mathrm{r}_{\mathrm{s}}=0.44, \mathrm{p}=0.05\right)$ and inversely correlated with the residual volume $(\mathrm{RV}) \%$ pred $\left(r_{s}=-0.56, p=0.02\right)$. The extent of adventitial inflammation $(\% \mathrm{AI})$ correlated with the forced vital capacity (FVC) $\%$ pred $\left(r_{s}=0.48, p=0.03\right)$. In those patients whose FEV1 was $<60 \%$ pred $(n=9)$, subepithelial fibrosis correlated with AF $\left(r_{s}=0.68, p=0.04\right)$; AF correlated with FEV1 ( $\mathrm{rs}=0.77, \mathrm{p}=0.01)$ and with FEV1/FVC $\left(r_{s}=0.75, p=0.02\right)$.

When comparing histological differences between patients with a normal or an obstructive spirometric pattern, the AIS was higher in those with normal spirometry (Mann-Whitney U test, $\mathrm{p}=0.04$ ).

No significant differences in pathological patterns were found between the patients who did or did not require lung transplantation.

\section{Imaging findings}

HRCT studies were available for 10 patients. Of those, eight had been performed within $1 \mathrm{yr}$ of the
Table 5. - Original pathological diagnoses of the cases in the study

Patient Original diagnosis

no.

$\begin{array}{ll}1 & \text { Obliterative bronchiolitis } \\ 2 & \text { Mild chronic bronchiolitis } \\ 3 & \text { Obliterative destructive bronchiolitis } \\ \text { consistent with rheumatoid arthritis } \\ 4 & \text { Mild chronic bronchiolitis } \\ 5 & \text { Mild chronic bronchiolitis, pulmonary } \\ \text { vascular remodelling with intimal } \\ \text { thickening, normal lung parenchyma } \\ \text { Multifocal interstitial fibrosis consistent } \\ \text { with bronchiolitis obliterans. Giant cells } \\ \text { and cholesterol clefts can be the result } \\ \text { of a hypersensitivity pneumonitis } \\ \text { Mild chronic bronchiolitis with centrilobular } \\ \text { emphysema }\end{array}$

8 Chronic bronchiolitis, intraalveolar haemorrhage

9 Patchy nonspecific lymphocytic pneumonitis with lymphocytic bronchiolitis. Poorly formed granulomas. Differential diagnosis includes hypersensitivity pneumonitis, post-viral infection, collagen vascular disease.

Concentric, partially obliterative, active bronchiolitis

Constrictive bronchiolitis with focal bronchiolar fibrous obliteration, lymphocytic infiltrate and peripheral emphysema

12 Chronic obliterating bronchiolitis

13 Bronchiolitis obliterans with mucoid impaction. Many neutrophils inside the mucus plugs. Multifocal ulceration of bronchial epithelium, with formation of granulation tissue polyps within the lumens of muscular bronchioles. Consistent with previous viral infection.

14 Destructive obliterative bronchiolitis with focal areas of mucus plugging

15 Mild chronic bronchiolitis with smooth muscle hypertrophy and irregular fibrosis with luminal distortion of respiratory and terminal bronchioles.

16 Obliterative bronchiolitis

17 Chronic bronchiolitis of the constrictive type (sometimes called small airways disease)

18 Proliferative obstructive bronchiolitis, pulmonary haemosiderosis

19 Mild chronic bronchiolitis

biopsy. In five cases, expiratory images were also available. Bronchial-wall thickening was present in five cases, bronchiectasis in two cases, and bronchiolectasis in one case. No patient had tree-in-bud findings. Centrilobular nodules were observed in two patients. A ground-glass pattern was relatively common, being observed in seven patients. Inspiratory air-trapping was present in seven cases, whereas expiratory airtrapping was found in all five cases where expiratory scans were available. Because of the small number of available studies, no statistical analysis was performed.

\section{Analysis according to smoking status}

No differences related to smoking status (smoker, former-smoker, never-smoker) were found in spirometry 
Table 6. - Bronchiolar inflammation

\begin{tabular}{lcccc}
\hline Site of inflammation & No of patients & Grade of inflammation & Bronchioles involved \% & Total inflammation score \\
\hline Subepithelial & $19 / 19$ & $2(1-2)$ & $60(22.5-71.5)$ & $86.8(27.8-140.3)$ \\
Adventitial & $19 / 19$ & $1.5(1.2-1.8)$ & $54(46.3-90.5)$ & $89.6(58.3-123.2)$ \\
\hline
\end{tabular}

Data are presented as median (confidence interval).

or pathology (Kruskal-Wallis one-way analysis of variance).

\section{Symptom duration and spirometry}

The duration of dyspnoea was inversely correlated with $\mathrm{FEV} 1 / \mathrm{FVC}\left(\mathrm{r}_{\mathrm{s}}=-0.49, \mathrm{p}=0.04\right)$ and mid-expiratory flow $(\mathrm{MEF}) \%$ pred $\left(\mathrm{r}_{\mathrm{s}}=-0.69, \mathrm{p}=0.03\right)$, and positively correlated with $\mathrm{RV} \%$ pred $\left(\mathrm{r}_{\mathrm{s}}=0.61, \mathrm{p}=0.01\right)$.

\section{Follow-up}

After lung biopsy, patients were treated with prednisone, and/or cyclophosphamide or azathioprine or colchicine or clarithromycin. Four (patients 1, 3, 6, 11) underwent lung transplantation, and remain alive. Six improved symptomatically and spirometrically on medical therapy (patients $5,8,9,13,14,15)$, one (patient 12) is stable and one (patient 16) died shortly after consultation at the National Jewish Center. Six are alive but there was no available clinical follow-up. Patient 2 was lost to follow-up. HRCT follow-up was not available for most of the patients.

\section{Discussion}

The cases studied were selected on the basis of pathological criteria of bronchiolar inflammation and scarring. Despite the common pathology, there was a marked heterogeneity in their clinical presentation and radiological and functional features.

Studies addressing whether there is a close relationship between the histological lesions in obliterative bronchiolitis and the patients' physiological impairment are still lacking. Prior to the present study, the authors hypothesized that indices of disease severity and airflow limitation would correlate with bronchiolar fibrosis. It was found that the cases of obliterative bronchiolitis presented in this study fitted the description provided by COLBY and MYERs [12], where small airways disease were divided into two groups: 1) cases with acute and chronic cellular bronchiolitis with less conspicuous scarring; and 2) cases with constrictive

Table 7.-Bronchiolar collagen deposition

\begin{tabular}{lcl}
\hline Type of collagen deposition & $\begin{array}{c}\text { No of } \\
\text { patients }\end{array}$ & $\begin{array}{c}\text { Bronchioles } \\
\text { involved \% }\end{array}$ \\
\hline Subepithelial deposition & $15 / 19$ & $29(5.3-55)$ \\
Adventitial deposition & $12 / 19$ & $16(0-33.3)$ \\
Minimal collagen deposition & $16 / 19$ & $21(6.5-29.6)$ \\
\hline
\end{tabular}

Data are presented as median (confidence interval). bronchiolitis, varying from fibrotic and inflammatory lesions to complete airway obliteration. CoLBY and Myers [12] commented that the most common findings were mild chronic inflammatory infiltrates and mural scarring. In the present study, it was found that subepithelial fibrosis inversely correlated with subepithelial and adventitial inflammation. These findings indicate that there is a temporal compartmentalization between the inflammatory and fibrotic bronchiolar changes. In other words, the subepithelial bronchiolar lesions were either primarily fibrotic or inflammatory. This implies that inflammation and fibrosis occur at different time points in the course of the disease, or alternatively, that patients with one type of presentation usually do not show transition to another type of bronchiolar pathology.

Surprisingly, no correlation was found between the degree of subepithelial collagen deposition and airflow indices, for the entire group, or the obstructive subgroup. The lack of significant correlation between morphological parameters and airway physiology in the present study may have occurred because chronic bronchiolitis presents as a heterogeneous disease at the lung tissue level. Inflammation was mild or moderate and involved only half of the bronchioles. Moreover, SF was present in only one-third of the bronchioles examined. Importantly, four patients did not have morphological evidence of $\mathrm{SF}$, despite the clinical evidence of airflow-limitation. AF affected a much smaller number of airways.

A clinical diagnosis of asthma was common in the patient group. Pathological studies in asthma usually focus on bronchial alterations, including smooth muscle cell hyperplasia, mucus plugging, thickening of the epithelial basement membrane, submucosal oedema, infiltration of eosinophils, mast cells and lymphocytes, and epithelial cell shedding. Most of the papers published on the morphology of small airways in asthma reveal an increased thickness of the basement membrane, the smooth muscle layer, the adventitial area and the submucosa. Distal-airway inflammation is also present. In addition, SAETTA et al. [14] observed luminal obstruction by mucus in $50 \%$ of the bronchioles examined in six patients who died suddenly during an asthma attack. However, evidence of luminal narrowing is scarce. In most of the studies, irreversible scarring of small airways has not been specifically addressed. In a study of six elderly nonsmoking asthmatics who died of causes other than asthma, SoBOnya [15] found two cases with reduced small airways diameter, with histological evidence of inflammation or fibrosis. Moreover, there have been no detailed pathological studies of lungs with refractory asthma including a component of irreversible airway remodelling [16]. Despite the bronchodilator 
response in several of the present study's patients, their pathology was not consistent with this disease. It is possible that some cases of severe, progressive asthma actually represent obliterative bronchiolitis. Occupational exposure to isocyanate compounds can cause asthma or hypersensitivity pneumonitis [17]. Biopsies of patients with isocyanate-induced asthma have similar findings as those described above [18]. Patient 8, who was exposed to isocyanates, had a clinical disease consistent with asthma, with considerable bronchodilator response. Biopsy revealed chronic bronchiolitis with basement membrane thickening and peribronchiolar scarring, as well as haemosiderinladen macrophages. Smooth-muscle hyperplasia and eosinophils were not noted. This patient's lung function had improved, but oral corticosteroid therapy is still required.

Exposures to either nitrous oxides [11], sulphurous oxides or ammonia have been associated with the development of obliterative bronchiolitis [19]. The typical clinical course following toxic fume exposure consists of three phases: 1) an acute onset, with upper respiratory symptoms and, sometimes, pulmonary oedema; 2) a latent period; and 3) an irreversible obstructive, mixed or restrictive physiological picture. Patient 15 had continuous symptoms of dyspnoea and cough after his fume exposure. Physiological tests at the time of lung biopsy, 4 months after exposure, indicated normal airflow parameters with a decreased $\mathrm{RV}$ and marked arterial oxygen desaturation during exercise. Although the patient did not have obstructive physiology, pathological findings demonstrated obliterative bronchiolitis. After 4 yrs of treatment with combinations of prednisone, cyclophosphamide and colchicine, the patient now has normal spirometry, except for increased RV and a normal diffusing capacity and exercise capacity.

Constrictive bronchiolitis is a well known complication of rheumatoid arthritis [20]. Gold and penicillamine therapy have been implicated in some patients [21]. The precipitating factor is unclear in patient 3, who had received both of these treatments prior to onset of symptoms.

Based on the lung biopsy, three patients might have had some of the histological findings of hypersensitivity pneumonitis. This disease is histologically characterized by a uniform chronic interstitial pneumonia with peribronchiolar accentuation, non-necrotizing granulomas in the peribronchiolar interstitium, and foci of bronchiolitis obliterans with organizing pneumonia [22]. None of the present cases met all these diagnostic criteria. Since not all cases exhibit the full range of histological findings, the possibility of hypersensitivity pneumonitis cannot definitely be excluded in these three patients. Moreover, constrictive bronchiolitis has been described in association with hypersensitivity pneumonitis [12]. However, overt severe airflow obstruction is considered rare [23].

Patient 18 had a p-ANCA titre of $1: 10,240$ and had had a cough, without dyspnoea, since early childhood. Eight years prior to biopsy, this patient developed exertional dyspnoea and was diagnosed as having asthma, and treated with inhaled bronchodilators and corticosteroids, as well as short bursts of oral corticosteroids. Lung function deteriorated rapidly, demonstrating airflow limitation. Prior to lung biopsy, the patient experienced migratory arthralgias, anorexia, weight loss and anaemia. The renal function remained normal. The patient was treated with prednisone and trimethoprim-sulphamethoxazole for exacerbation of respiratory symptoms, and showed improvement in both symptoms and spirometry. The biopsy showed inflammation and destruction of the respiratory bronchioles with fibrotic changes extending from them. Haemosiderin-laden macrophages were present in the alveolar spaces. Intra- and extracellular deposition of immunoglobulin (Ig)A, IgM and $\mathrm{C}_{3}$ was demonstrated by immunofluorescence. The patient was placed on cyclophosphamide, with resolution of her systemic symptoms. Respiratory function stabilized, but did not return to normal. In a report of histological changes in the lung in 14 patients with MPO-ANCA, no patients were found with nongranulomatous airway inflammation [24]. A recent study described MPO-ANCA positivity in four of 30 patients with diffuse panbronchiolitis [25]. The patient's history of bronchitic symptoms since childhood is suggestive of diffuse panbronchiolitis, however the patient never had sinusitis. Furthermore, biopsy did not reveal the typical foamy interstitial macrophages seen in diffuse panbronchiolitis. Conversely, SchwARz et al. [26] have reported two cases of microscopic polyarteritis with recurrent pulmonary capillaritis and diffuse alveolar haemorrhage, which developed progressive irreversible airflow limitation 10 yrs after disease onset.

Chest radiographical findings in obliterative bronchiolitis consist of decreased vascular markings and hyperinflation. The HRCT scan shows areas of decreased lung attenuation (air trapping) interspersed with areas of normal attenuation during expiration and often during inspiration. Bronchiectasis, bronchial-wall thickening or bronchiolar dilatation may also be present [27]. The same constellation of findings was present in the present patients. However, seven of 10 patients also had areas of ground-glass attenuation, which was relatively mild in extent. The present authors have previously identified ground glass attenuation in $50 \%$ of patients with constrictive bronchiolitis [28]. The appearance of ground glass attenuation may be related to relative over-perfusion of more normal areas of lung.

The classification of inflammation as submucosal or adventitial was based on the description of MYERS and Colby [29] of the inflammation in constrictive bronchiolitis as being intraluminal, mucosal, submucosal and peribronchiolar. Conversely, Gosink et al. [5], in an earlier report, had classified bronchiolar collagen deposition in a similar manner, characterizing the fibrosis found in constrictive bronchiolitis as submucosal and peribronchiolar. To the best of the authors' knowledge, the classification proposed in the present study has not appeared in any research papers on bronchiolitis. However, HaLey et al. [30] have utilized a similar approach in a morphometric study of the distribution of CD45+ leukocytes and eosinophils in patients who died with severe asthma. The airway wall was studied as two separate 
compartments, an "inner area" (between basement membrane and smooth muscle) and an "outer area" (between smooth muscle and alveolar attachments). The inner and outer areas correspond to the subepithelial and adventitial partitioning respectively in the present study.

Approximately one-half of the patients in the present study were current or former smokers. Comparing the three groups (never-smokers, former smokers, current smokers), there were no significant differences in lung function or pathology between them. In addition, pack-yrs of smoking and age could not explain either the degree of airflow limitation or patients' symptoms. This implies that the bronchiolar lesions in the current smoking group were probably not related to tobacco use. Diffusing capacity for $\mathrm{CO}$, corrected for alveolar volume, was normal in the present patient group, excluding any extensive interstitial involvement. Surprisingly, there were no significant differences in the pathological findings between the four patients who required lung transplantation and those that did not. The small number of patients requiring transplantation might account for this finding.

In summary, nineteen patients with chronic bronchiolitis, with or without luminal obliteration, were studied. It appears that there is temporal and spatial compartmentalization of the bronchiolar inflammation and collagen deposition in these patients, particularly between the subepithelial and adventitial region of the bronchioles. Only adventitial inflammation was clearly associated with preserved lung function. Further studies are needed to clarify the complex histological category of obliterative bronchiolitis.

\section{References}

1. Lange W. Ueber eine eigenthumliche Erkrankung der kleinen Bronchien und Bronhilen (Bronchitis und Bronchiolitis obliterans). Dtsch Arch Klin Med 1901; 70: 324-364.

2. Blumgart HL, MacMahon HE. Bronchiolitis fibrosa obliterans: a clinical and pathologic study. Med Clin North Am 1929; 13: 197-214.

3. Baar HS, Galindo J. Bronchiolitis Fibrosa Obliterans. Thorax 1966; 21: 209-214.

4. Macklem PT, Thurlbeck WM, Fraser RG. Chronic obstructive disease of small airways. Ann Intern Med 1971; 74: 167-177.

5. Gosink BB, Friedman PJ, Liebow AA. Bronchiolitis obliterans. Roentgenologic-pathologic correlation. Am J Roentgenol 1973; 117: 816-832.

6. Turton CW, Williams G, Green M. Cryptogenic obliterative bronchiolitis in adults. Thorax 1981; 36: 805-810.

7. Guerry-Force ML, Muller NL, Wright JL. A comparison of bronchiolitis obliterans with organizing pneumonia, usual interstitial pneumonia, and small airways disease. Am Rev Respir Dis 1987; 135: 880-884.

8. Kindt GC, Weiland JE, Davis WB, et al. Bronchiolitis in adults. A reversible cause of airway obstruction associated with airway neutrophils and neutrophil products. Am Rev Respir Dis 1989; 140: 483-492.

9. Edwards C, Cayton R, Bryan R. Chronic transmural bronchiolitis: a non-specific lesion of small airways. J Clin Pathol 1992; 45: 993-998.

10. Kraft M, Mortenson RL, Colby TV, Newman L, Waldron JA, King TE. Cryptogenic constrictive bronchiolitis. A clinicopathologic study. Am Rev Respir Dis 1993; 148: 1093-1101.

11. King TE. Bronchiolitis. In: Schwarz MI, King TE. Interstitial Lung Disease. 3rd ed. Hamilton, Ontario, B.C. Decker, 1998; pp. 685-696.

12. Colby TV, Myers JL. Clinical and histologic spectrum of bronchiolitis obliterans, including bronchiolitis obliterans organizing pneumonia. Semin Respir Med 1992; 13: 119-133.

13. Cool CD, Stewart JS, Werahera P, et al. Threedimensional reconstrunction of pulmonary arteries in plexiform pulmonary hypertension using cell-specific markers. Evidence for a dynamic and heterogeneous process of pulmonary endothelial cell growth. Am J Path 1999; 155: 411-419.

14. Saetta M, DiStefano A, Rosina C, Thiene G, Fabbri LM. Quantitative structural analysis of peripheral airways and arteries in sudden fatal asthma. Am Rev Respir Dis 1991; 143: 138-143.

15. Sobonya R. Quantitative structural alterations in long-standing allergic asthma. Am Rev Respir Dis 1984; 130: 289-292.

16. Anonymous. Proceedings of the ATS Workshop on Refractory Asthma. Am J Respir Crit Care Med 2000; 162: 2341-2351.

17. Montanaro A. Isocyanate asthma. In: Bardana EJ, Montanaro A, O'Hollaren MT. Occupational Asthma. Philadelphia, PA, Hanley and Belfus, 1992; pp. 131143.

18. Mapp CE, Saetta M, Maestrelli P, et al. Mechanisms and pathology of occupational asthma. Eur Respir $J$ 1994; 7: $544-554$.

19. Wright JL, Cagle P, Churg A, Colby TV, Myers J. Diseases of the small airways. Am Rev Respir Dis 1992; 146: 240-262.

20. Geddes D, Corrin B, Brewerton D. Progressive airflow obliteration in adults and its association with rheumatoid arthritis. Q J Med 1977; 46: 427-444.

21. Holness I, Tenebaum J, Cooter NB, Grossman RF. Fatal bronchiolitis obliterans associated with chrysotherapy. Ann Rheum Dis 1983; 42: 593-596.

22. Immunologic lung disease. Katzenstein A-L A. Katzenstein and Askin's surgical pathology of nonneoplastic lung disease. 3rd ed. Philadelphia, PA, W.B.Saunders Company, 1997; pp. 139-167.

23. Selman-Lama M, Perez-Padilla R. Airflow obstruction and airway lesions in hypersensitivity pneumonitis. Clin Chest Med 1993; 14: 699-714.

24. Gaudin PB, Askin FB, Falk RJ, Jennette JC. The pathologic spectrum of pulmonary lesions in patients with anti-neutrophil cytoplasmic autoantibodies specific for anti-proteinase 3 and anti-myeloperoxidase. Am J Clin Pathol 1995; 104: 7-16.

25. Sugiyama Y, Kitamura S. Antineutrophil cytoplasmic antibodies in diffuse panbronchiolitis. Respiration 1999; 66: 233-235.

26. Schwarz MI, Mortenson RL, Colby TV, et al. Pulmonary capillaritis. The association with progressive irreversible airflow limitation and hyperinflation. Am Rev Respir Dis 1993; 148: 507-511.

27. Hansell DM, Rubens MB, Padley SPG, Wells AU. Obliterative bronchiolitis: individual CT signs of small 
airways disease and functional correlation. Radiology 1997; 203: 721-726.

28. Jensen SP, Lynch DA, Brown KK, Wenzel SE, Newell JD Jr. High resolution CT features of severe asthma and bronchiolitis obliterans. Radiology 2000; 217: 595.

29. Myers JL, Colby TV. Pathologic manifestations of bronchiolitis, constrictive bronchiolitis, cryptogenic organizing pneumonia, and diffuse panbronchiolitis. Clin Chest Med 1993; 14: 611-621.

30. Haley KJ, Sunday ME, Wiggs BR, et al. Inflammatory cell distribution within and along asthmatic airways. Am J Respir Crit Care Med 1998; 158: 565-572. 